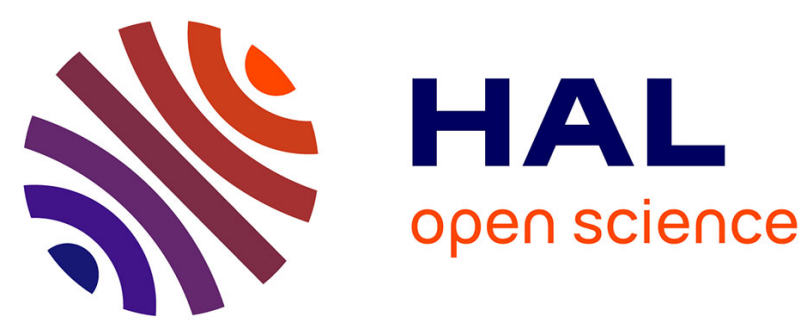

\title{
Importance of Synchronizing Radars with V2X communication for Radar Interference Mitigation
}

Sylvain Roudiere, Vincent Martinez, Daniel Delahaye

\section{To cite this version:}

Sylvain Roudiere, Vincent Martinez, Daniel Delahaye. Importance of Synchronizing Radars with V2X communication for Radar Interference Mitigation. 2021 IEEE Intelligent Transportation Systems Conference (ITSC), Nov 2021, Indianapolis, United States. 10.1109/itsc48978.2021.9565081 . hal03480272

\section{HAL Id: hal-03480272 \\ https://hal-enac.archives-ouvertes.fr/hal-03480272}

Submitted on 31 Dec 2021

HAL is a multi-disciplinary open access archive for the deposit and dissemination of scientific research documents, whether they are published or not. The documents may come from teaching and research institutions in France or abroad, or from public or private research centers.
L'archive ouverte pluridisciplinaire HAL, est destinée au dépôt et à la diffusion de documents scientifiques de niveau recherche, publiés ou non, émanant des établissements d'enseignement et de recherche français ou étrangers, des laboratoires publics ou privés. 


\title{
Importance of Synchronizing Radars with V2X communication for Radar Interference Mitigation
}

\author{
Sylvain Roudiere ${ }^{12 *}$, Vincent Martinez ${ }^{2}$, Daniel Delahaye ${ }^{3}$ \\ ${ }^{1}$ ANITI, Université Fédérale Toulouse Midi-Pyrénées, Toulouse, France \\ ${ }^{2}$ NXP, Semiconductors, Toulouse, France \\ ${ }^{3}$ École Nationale de l'Aviation Civile, Toulouse, France \\ * Corresponding email: sylvain.roudiere@univ-toulouse.fr
}

\begin{abstract}
As the number of vehicles equipped with radars sensors is rapidly increasing, the risk of harmful interference is increasing, especially since the radar waveform parameters are not regulated. Interference mitigation techniques are becoming important for radars to operate properly in this complex environment. We presented in a previous study that the use of V2X technology to communicate radars physical properties as well as the parameters of their waveform allows for new anticipation strategies. These strategies focus on identifying potential interferers thanks to $\mathrm{V} 2 \mathrm{X}$ communications and adapt the radar waveform to minimize the risk of interference. This article investigates the importance of time synchronization between the radar's waveform and the V2X messages emissions when using such a cooperative strategy.
\end{abstract}

\section{INTRODUCTION}

Advanced Driver Assistance Systems (ADAS) is a growing trend in the automotive domain. As stated by [1] and [2], the use of ADAS will keep growing for the years to come as the proportion of cars on the road equipped with radars will reach $50 \%$ ( $\approx 700$ million cars) according to their estimations. This rapid increase in the number of radars will lead to an increased risk of harmful interference between radars (from radar blindness to false targets) as more and more radars will need to efficiently share the $76-81 \mathrm{GHz}$ bandwidth. Even though some studies [3] suggest that sharing this bandwidth between short (SRR) and long (LRR) range radars will lead to saturating interference from LRRs to SRRs, radar manufacturers have little regulatory obligations and can deploy radars that use any kind of waveform, anywhere within the limits of the $76-81 \mathrm{GHz}$ band.

As presented in our previous work [4], coordinating the use of the bandwidth with the V2X technology has a lot of potential. V2X technology allows a Cooperative Intelligent Transport Systems (C-ITS) network to be established between actors on the road (vehicles, pedestrians, infrastructures, etc.). An example of C-ITS currently being deployed is the ETSI
ITS network ${ }^{1}$. It is used in the new Volkswagen Golf 8 cars [5], or also in connected infrastructures in Austria [6], just to name a few. Multiple types of messages are defined for ITS applications, we focus in our work on the cooperative awareness messages (CAM) [7] which is used to convey information about the vehicle such as coordinates, speed and heading, as well as multiple optional and customizable fields. A detailed description of the different fields available in a CAM is presented in Figure 1.

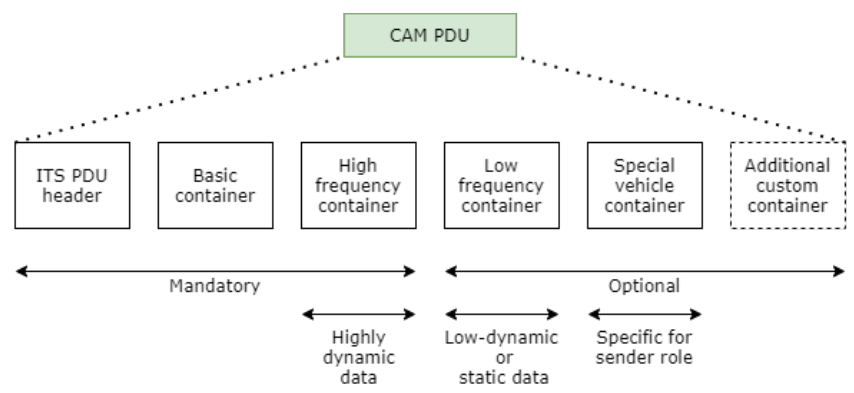

Fig. 1: A CAM consists of a collection of data elements, arranged in a hierarchical order. The Vehicle ID in the header, then basic data like timestamp and position, then a container for highly dynamic data such as speed, heading, acceleration and curvature. Then, optional containers for low-dynamic and static data such as the vehicle role, category or basic sensors and finally and container for the vehicle category details (public transport, rescue).

The goal of this paper is to present the importance of time synchronizing the waveform changes of automotive radars with the V2X messages emissions in order for V2X based interference mitigation strategies to work to their full potential.

The paper is organized as follows. Section II provides a brief overview of description of the radar interference principles, Section III presents in more detail the V2X

${ }^{1}$ The European Telecommunications Standards Institute (ETSI) aims with Intelligent Transportation Systems (ITS) to provide services relating to different modes of transport and traffic management to make more coordinated, safer and smarter use of transport networks. https://www. etsi.org/technologies/automotive-intelligent-transport\#mytoc3 gives an introduction to ETSI and the ITS Communications Architecture is available at https://www.etsi.org/deliver/etsi_en/302600_302699/302665/01.01.01_60/ 1 en_302665v010101p.pdf. 
technology, followed in Section IV by the process used to simulate and evaluate its use for interference mitigation. Finally, Section V will present the results obtained with these evaluations, followed by our conclusion in Section VI.

\section{RADAR INTERFERENCE PRINCIPLE}

This study assumes the use of Frequency Modulated Continuous Waveform (FMCW) as it is known for its good performance in range and accuracy and is still the most commonly used radar waveform even decades after the first papers such as [8] [9] just to name a few. Other waveforms such as Phase Modulated Continuous Wave (PMCW) or Orthogonal Frequency-Division Multiplexing (OFDM) signals are not considered presently, but the concepts and results shown in this study should be applicable to any kind of waveform.

A FMCW signal is constructed by sending a sinusoidal signal whose frequency varies linearly with time from a start frequency $f_{c}$ to an end frequency $f_{c}+B$. This forms what is called a "chirp". This chirp pattern is then repeated hundreds of time in a row, with small pauses between chirps. After that, some processing time, defined by the duty cycle, is needed to compute the data gathered by the antenna. The combination of chirps and processing time is called a frame.

When a chirp is emitted, the signal will be reflected by the targets that are in the Field of View (FoV) of the radar and the echoes are then detected by the receiver. From the delay and the phase of the received echoes, range and velocity of targets can be determined.

During a chirp emission, as the receiver is listening for the echo of a target, it can detect other signals whose frequencies are within the receiver bandwidth. The correlation between the radar's own signal and the interferer's one will impact the effect of the interference on the system, as illustrated in Figure 2.

When dealing with a low correlated interferer, an increase of the noise floor can be observed for the victim radar. The noise floor increase will be more important as the proportion of the chirp that is interfered increases. This kind of interference reduces the maximum range of the radar, as the power of previously detectable echoes might be below the new increased noise floor, leading to total radar blindness in the worst-case scenario.

In the case of a perfectly correlated interferer, since the interferer's signal resembles a regular echo from a target, the interference will be interpreted as a regular target. Since this target doesn't exist, we call them false targets. These can sometimes be filtered on the application level as they might not be present on every chirp or frame.

\section{C-ITS AND V2X TECHNOLOGY}

Cooperative Intelligent Transport Systems (C-ITS) provide a flexible framework for vehicles, VRU (Vulnerable Road Users) and infrastructure to share information. This network is using the $5.9 \mathrm{GHz}$ ITS band and is organized with various layers (Access layer, Facilities and Applications layers). The formatting and contents of the messages is strictly defined to ensure inter-operability, for example of the CAM (Cooperative Awareness Messages) for ETSI ITS. Figure 3 depicts a simplified representation of the ETSI ITS architecture. All ETSI ITS standards at Facilities and Application layers are access layer technology agnostic.

Today, two distinct options are available for the access layer of such networks, namely ETSI ITS-G5 ${ }^{2}$ based on the IEEE 802.11p access layer and 3GPP C-V2X based on LTEV2X mode 4 or $5 \mathrm{G}$ NR V2X.

The key principle of $\mathrm{V} 2 \mathrm{X}$ communication are summarized as follow.

- Broadcast transmission: V2X messages are sent in broadcast mode and thus directed to all participants within the covering range;

- Omni-directional: V2X systems operating at $5.9 \mathrm{GHz}$ have omni-directional antenna patterns;

- Distributed scheduling: adhoc V2X networks have no "master" orchestrator and nodes coordinate their messages scheduling without infrastructure needs (e.g. cellular base-stations are not required in such networks);

- Typical communication range: several V2X field tests [10] [11] demonstrated a much higher maximum achievable distance, between 1000 and 1400 meters.

- Typical transmission rate: 1-10 Hz rate, depending on vehicle dynamics [7]

The performance of V2X networks is measured in terms of Packet Reception Ratio (PRR) versus distance and in terms of End-to-End Delay (EED), for which a statistical distribution is used. Numerical simulations of LTE-V2X and IEEE $802.11 \mathrm{p}$ access layers have been conducted to extract the data in figure 4 and 5 which are respectively the figures of merit of the PRR and the EED. For this study, the simulator LTEV2Vsim version 5.2.5 has been used ${ }^{3}$. Reference V2X PRR and EED curves used for the present study are shown in below figure.

\section{Evaluation}

\section{A. Simulation environment}

To evaluate the effect of V2X-Radar time synchronization on the performance of $\mathrm{V} 2 \mathrm{X}$ based interference mitigation strategies, we use a simulator that we developed in Python. This environment simulates the interactions between the different aspects of an automotive scenario using V2X and

\footnotetext{
${ }^{2}$ ITS-G5 defines a protocol stack for vehicular communications in an ad-hoc network to be used in the $5,9 \mathrm{GHz}$ frequency band allocated in Europe. Its access layer is based on IEEE 802.11p standard. The ITS-G5 standard adds features for decentralized congestion control (DCC) to control the network load and avoid unstable behavior.

${ }^{3}$ LTEV2Vsim is an open-source simulator developed by the Italian CNIT, CNR-IEIIT institute and the University of Bologna [12] and used in various technical studies such as [13]. CAM message length of 350 bytes is used, 2 according to average CAM size observed in real-life recorded traces [14]
} 

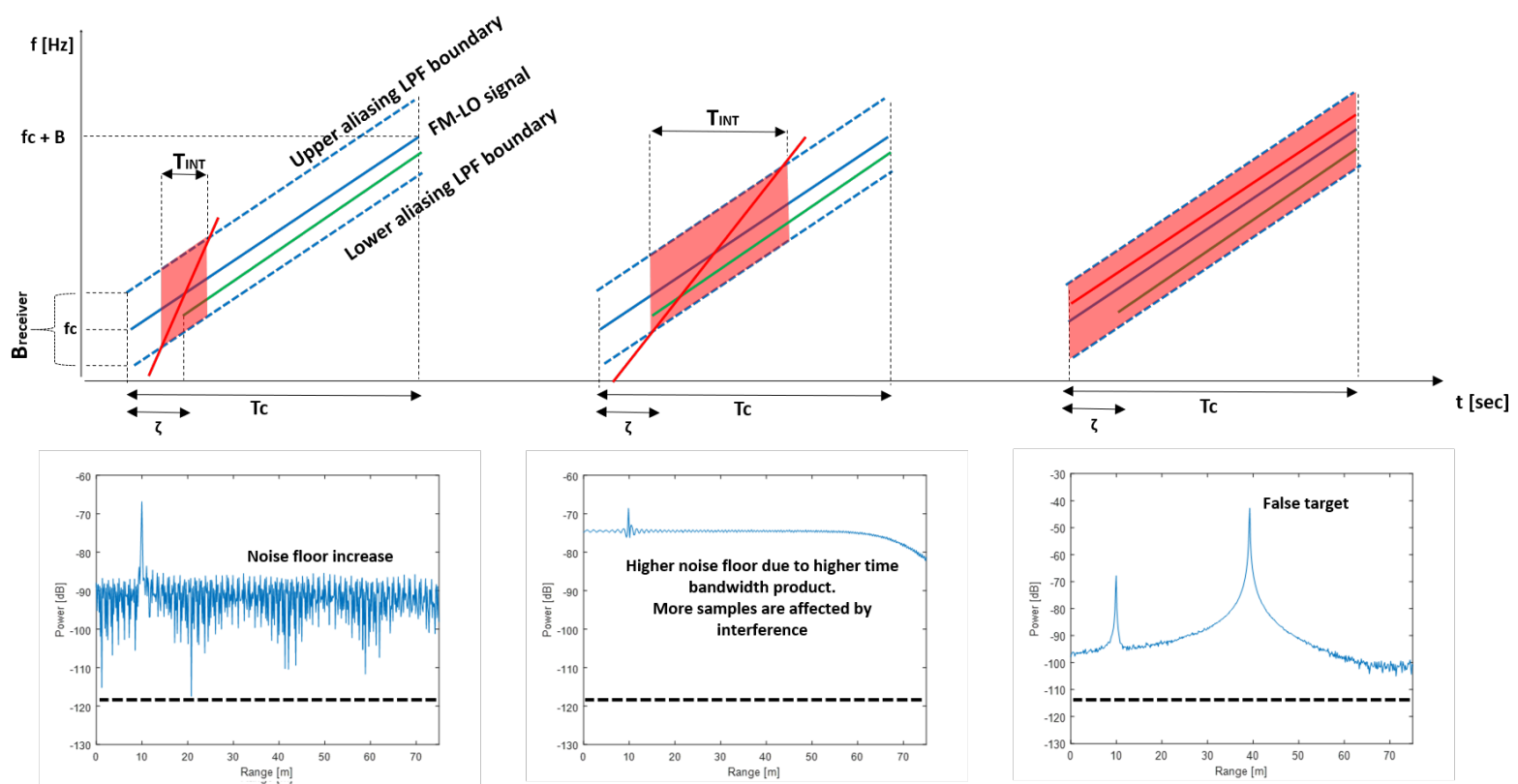

Fig. 2: On the top row, multiple examples of interference between a victim sending a FMCW chirp in blue waiting for the echo in green and an interferer signal in red crossing the victim's receiver bandwidth. On the bottom row, the impact of the interferer on the processed signal. The echo from the target is generating a power spike around $10 \mathrm{~m}$ at $-67 \mathrm{~dB}$. The noise floor, usually at $-120 \mathrm{~dB}$ (dotted-line), will increase depending on the correlation of the interferer with the victim. From left to right: (1) The interfere in red has a low correlation with the victim in blue, leading to a relatively small increase of the noise floor as few samples are affected. (2) As the correlation increases, the noise floor increases even more, leading to a potential blindness of the radar. (3) In the case of perfect correlation, the interference can be interpreted as a target as it resembles an echo of the blue signal with a random delay. The power associated with this false target may be much higher than a regular target since it doesn't necessarily has lost power due to reflection compared to the echo from a target.

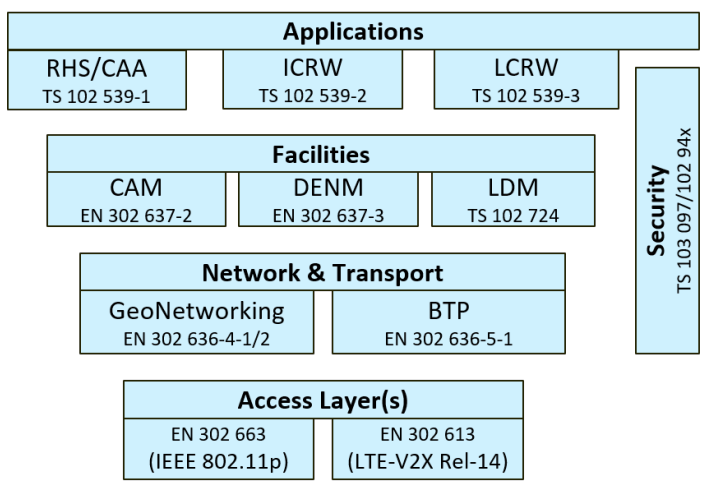

Fig. 3: Depiction of the ETSI ITS Architecture from a high-level view. CAMs are generated at the Facilities level and triggered by drive dynamics. They are encapsulated into GeoNetworking messages and transferred via Basic Transport Protocol (BTP) to the access layer. All ETSI ITS standards at Facilities and Application layers are access layer technology agnostic, but today, the two options available for the access layer are ETSI ITS-G5 based of IEEE 802.11p and 3GPP C-V2X based on LTE-V2X Rel-14.

radars. It comprises three layers and takes a traffic as input.

To model the vehicles traffic, we have used the open source SUMO software ${ }^{4}$. This provides input traces to our model, containing position, speed and orientation of the vehicles.

\footnotetext{
${ }^{4} \mathrm{SUMO}$ is an open-source, highly portable and continuous multimodal traffic simulation package designed to handle large road networks and is used in many V2X studies. The software is available at https://www.eclipse.org/sumo/
}

The first layer is the V2X one. It simulates the transmission of CAMs between vehicles. It is based on IEEE802.11p reference communication characteristics. The performance of the technology used are simulated using look-up tables generated from the simulations mentioned in Section III. They include references of End-to-End Delay (EED) and Packet Reception Ratio (RRP). CAMs are broadcasted by the vehicles on the V2X layer and contain the regular CAM data about the vehicle's ID, type, speed, direction and position. They also include additional information about the car's radars (position, direction, field-of-view, power) and their currently used waveform parameters.

The second layer is the processing one. It extracts the data contained in received CAMs and adds it to the vehicle's context. The "context" of a vehicle is a list of all other radars / cars it is aware of, thanks to the V2X communications. This layer also computes which radar is potentially in the line-of-sight by checking which radar is facing towards its own radar and taking into account that cars act as shields form direct line-of-sight. This precomputing of line-of-sight allows to filter out irrelevant information and facilitate the computation of the best waveform parameters when it is required. The radar parameters can be changed at any time if interference is detected and the changes will be applied to the next signal frame.

Finally, the Radar layer estimates the amount of interfer3 ence received by each radar and stores data about the kind 


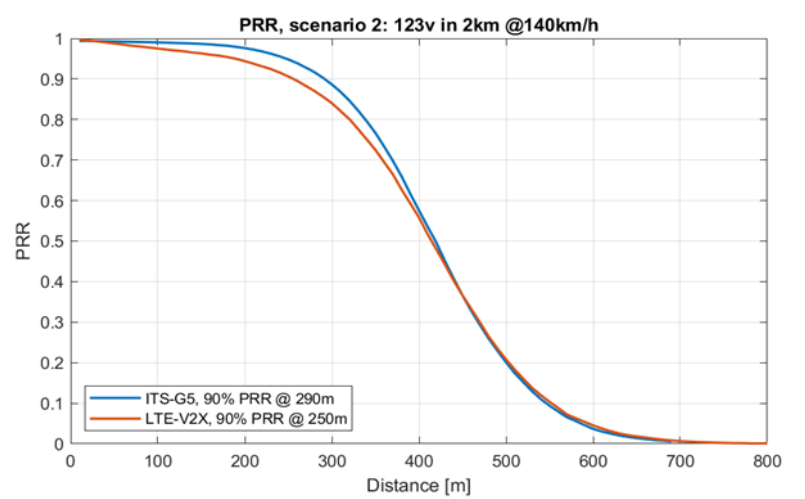

Fig. 4: V2X reference PRR curves for ITS-G5 and LTE-V2X.

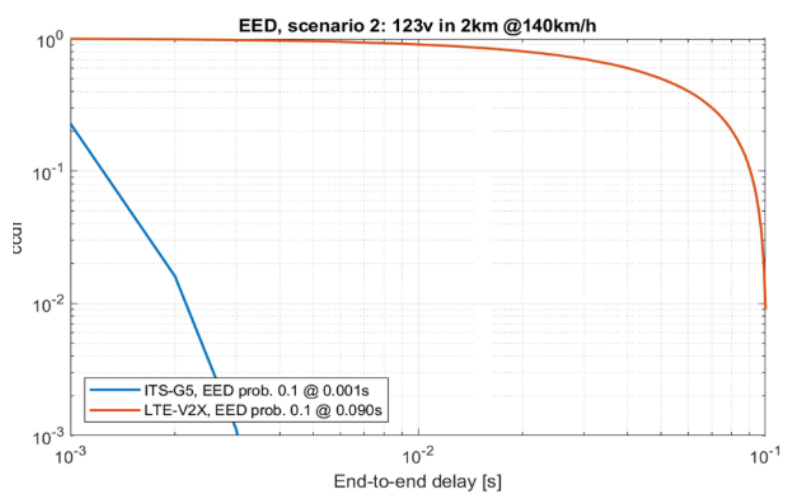

Fig. 5: V2X reference EED curves for ITS-G5 and LTE-V2X.

of signals used by each radar at any point in time.

The metric visualized to estimate the amount of interference received is the noise floor increase. The noise floor increase from an interfere is computed with the following formula:

$$
N F I=i n t_{\text {ratio }} *\left(n f-P_{\text {int }}\right)
$$

Where:

- $N F I$ is the noise floor increase in $\mathrm{dBm}$;

- int $_{\text {ratio }}$ is the percentage of the signal that is interfered with;

- $n f$ is the noise floor power without interference defined in the simulation $(-120 \mathrm{dBm})$;

- $P_{i n t}$ is power of the interference received by the victim's antenna.

\section{B. Simulation parameters}

The SUMO scenario used to evaluate the performances of our mitigation strategies is a $2.5 \mathrm{~km}$ highway scenario with $2 \times 3$ lanes. The layout can be seen in Figure 6. The upper side of the road has a group of 25 vehicles spread across $\approx 500 \mathrm{~m}$. Within this cluster of vehicles is located the vehicle from which we visualize the radar performances. The other side of the road has a continuous flow of vehicles with a density of $\approx 10$ vehicles per $100 \mathrm{~m}$.

Each car is equipped with a LRR, positioned on the front, pointing forward with a field-of-view of $20^{\circ}$ and a maximum range of $300 \mathrm{~m}$. The FMCW parameters used by the radars are selected from a list of 36 different sets of parameters

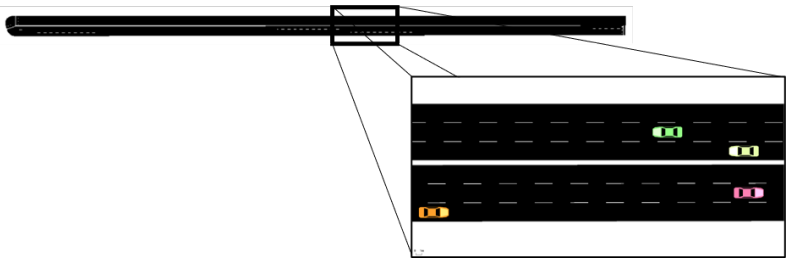

Fig. 6: SUMO layout: $2.5 \mathrm{~km}$ highway

that do not interfere with each other. The size of the list can be reduced to artificially increase the scenario's complexity. These sets of waveforms differ by their starting time and frequency in order not to overlap with each other. During the simulation, CAMs are generated at a constant frequency of $4 \mathrm{~Hz}$ (the average frequency for vehicles travelling at $55 \mathrm{kmph})$.

The mitigation strategy used by cars works as follow:

- If no interference is detected on the last frame, do nothing;

- Else, retrieve the list of potential interferers from the car's context;

- Retrieve which set of parameters is used by each of these radars;

- Select a set of parameters at random among the sets that are least used by potential interferers.

To evaluate the importance of synchronizing the radar changes with the V2X messages, we implemented three different ways to trigger the use of the mitigation strategy:

- V2X: right before sending a new CAM message;

- Mid-Interval-V2X: right in between the emission of two different CAM messages;

- Periodic-Uniform-Random (PUR): every $250 \pm 125 \mathrm{~ms}$;

- Frame: every frame.

Theses four methods can be visualized in Figure 7. The 'PUR' method serves as a baseline as the period at which radar parameters are changed is the same as the other two methods, but the delay between the change and the next CAM emission is random. The 'Frame' method is another baseline, as it represents the strategy of changing constantly the parameters until one without interference is found.

\section{RESULTS}

This section presents the averaged results from 300 simulations for each combination signal-synchronization type. Table I presents the average amount of noise floor increase (NFI) from the LRR of the observed vehicle as well as the average NFI during the 70 s to 140 s time window as it is where the two flows of vehicles are meeting (the most complex situation).

When the number of available sets of non-interfering parameters is set to 36 , we can notice better performances from the 'Frame' methods over the three others. The 'PUR', 'Mid-Interval-V2X' and 'V2X' methods all yield $\approx 50 \%$ 4 NFI. 


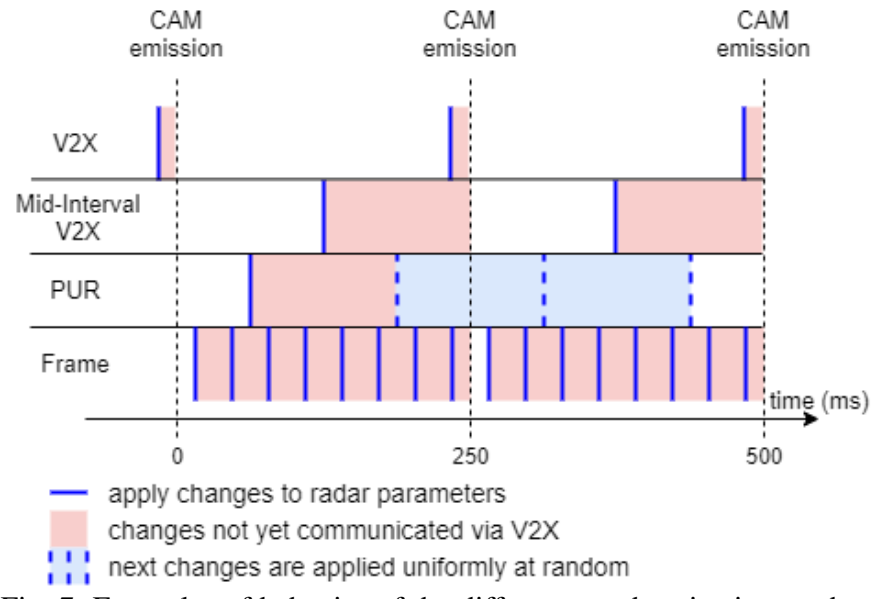

Fig. 7: Examples of behavior of the different synchronization methods. The 'V2X' method synchronizes the radar parameters changes (blue vertical line) with CAMs emissions, leading to a small time window during which other vehicles are not aware of the changes done (red area). 'Mid-Interval-V2X' changes the radar parameters right between the emission of two CAMs. Periodic-UniformRandom (PUR) doesn't synchronize with the CAM emission. The delay between two parameters changes is the CAM period \pm half the CAM period to keep the same average change frequency while being random. The 'Frame' method changes the parameters every signal frame (every $24 \mathrm{~ms}$ ).

At the peak traffic moment of the simulation, the observed radar has in its line-of-sight 40 other radars (directly or via the reflection from cars in front). Even when randomly choosing the set of parameters to use, the probability of choosing a set of parameters that no other radar in line-of-sight uses is $(35 / 36)^{40} \approx 32.4 \%$. During the $70-140$ s time frame, interference triggers on average between 2 and 3 changes of radar parameters every $100 \mathrm{~ms}$ across all radars. For this simulation that contains around 100 vehicles, that means that a radar is likely to change its parameters every 3 to 5 seconds. This stability implies that the context of the car is less likely to contain outdated information from other radars (the red area in Figure 7) when selecting new parameters. As a radar keeps its parameters for a long period of time, it is less likely for other radars to change their parameters between the time it decides to change its own and the next CAM containing the information of its change. In this kind of situation, the best way to mitigate interference is to adapt the radar parameters as fast as possible without taking into account the $\mathrm{V} 2 \mathrm{X}$ synchronization, that is why the 'Frame' method performs better, as they have the potential to change parameters more often than ' $\mathrm{V} 2 \mathrm{X}$ ', 'Mid-Interval-V2X' and 'PUR' in a short window of time. Indeed, even though the 3 methods have different timing or randomness, the average time between two changes of parameters still corresponds to the CAM emission period of $250 \mathrm{~ms}$, whereas the 'Frame' method can change every $\approx 25 \mathrm{~ms}$.

When increasing the complexity, we start to notice a difference between the different synchronization methods.
Increasing the probability of interference leads to more radars parameters changes per second, reducing the length of time where a CAM's information is valid while the outdated CAM data time frame is still the same. With only 18 sets of parameters available, the 'Mid-Interval-V2X' method yields a $15 \%$ higher noise floor than the 'V2X' one. The 'Frame' method also performs worse with a 3 times higher noise floor increase. The 'PUR' methods performance are still close to the ' $\mathrm{V} 2 \mathrm{X}$ ' one.

Finally, in the most complex scenario with only 12 sets of parameters available, the V2X-synchronized method yield better results than every other method. The "PUR" and "Mid-Interval-V2X" methods have respectively $32 \%$ and $47 \%$ higher noise floor increases, while the 'Frame' method yields the worst performances with a noise floor increase 2 times higher.

\begin{tabular}{|c|c|c|c|}
\hline Signal used & Synchronization used & NFI & $70-140 \mathrm{~s} \mathrm{NFI}$ \\
\hline \multirow{2}{*}{ FMCW } & Frame & $0.13 \mathrm{dBm}$ & $0.29 \mathrm{dBm}$ \\
alphabet & Mid-Interval-V2X & $0.21 \mathrm{dBm}$ & $0.45 \mathrm{dBm}$ \\
size: 36 & PUR & $0.18 \mathrm{dBm}$ & $0.41 \mathrm{dBm}$ \\
& V2X & $0.21 \mathrm{dBm}$ & $0.47 \mathrm{dBm}$ \\
\hline \multirow{2}{*}{ FMCW } & Frame & $3.49 \mathrm{dBm}$ & $8.47 \mathrm{dBm}$ \\
alphabet & Mid-Interval-V2X & $1.46 \mathrm{dBm}$ & $3.39 \mathrm{dBm}$ \\
size: 18 & PUR & $1.34 \mathrm{dBm}$ & $3.10 \mathrm{dBm}$ \\
& V2X & $1.29 \mathrm{dBm}$ & $2.97 \mathrm{dBm}$ \\
\hline \multirow{2}{*}{ FMCW } & Frame & $9.02 \mathrm{dBm}$ & $20.50 \mathrm{dBm}$ \\
alphabet & Mid-Interval-V2X & $6.12 \mathrm{dBm}$ & $14.07 \mathrm{dBm}$ \\
size: 12 & PUR & $5.49 \mathrm{dBm}$ & $12.64 \mathrm{dBm}$ \\
& V2X & $4.28 \mathrm{dBm}$ & $9.54 \mathrm{dBm}$ \\
\hline
\end{tabular}

TABLE I: Results for the three synchronization methods, extracted from simulation in Figure 8,9 and 10. For a low complexity of the environment, when the FMCW alphabet size is 36, the 'Frame' method performs better than the other three. The probability to pick a new set of parameters that none of the other radars in line-of-sight (LOS) is using is relatively high even when choosing at random. With 40 other radars in LOS, this probability is $(35 / 36)^{40} \approx 32.4 \%$. So quickly changing parameters yields better results than waiting to make the best change possible, as illustrated by the lower NFI from the 'Frame' method. For smaller alphabet sizes, this probability is much lower $\left((11 / 12)^{40} \approx 0.3 \%\right)$. Coordination between radars becomes necessary and the 'V2X' method guarantees that radars make choices with up-to-date data, yielding better results as the complexity of the environment increases. 'PUR' and 'Mid-Interval-V2X' methods yields intermediate results as they do not guarantee up-to-date data.

\section{CONCLUSION}

In this paper, we investigated the importance of synchronizing cars' radars parameters changes with the V2X communications when using an interference mitigation strategy based on V2X. As shown by the results of our simulations, time synchronizing the choice of radar parameters with the broadcasting of the message containing the information of the choice becomes a necessity when the radars are frequently changing their parameters. In stable situation where radars rarely receive interference, this synchronization isn't necessary. As the frequency at which radars are changing their parameters increases, the delay between the choice of new parameters and the emission 5 of the information starts to impact the overall amount of 
interference a radar could expect as more CAM's data is outdated.

A trade-off needs to be found between reacting quickly to an interference and keeping a certain stability to help other agents in their decision. Some method using V2X communication with coordination messages, or AI could be investigated in future works to automatically determine what is the best approach in each situation.

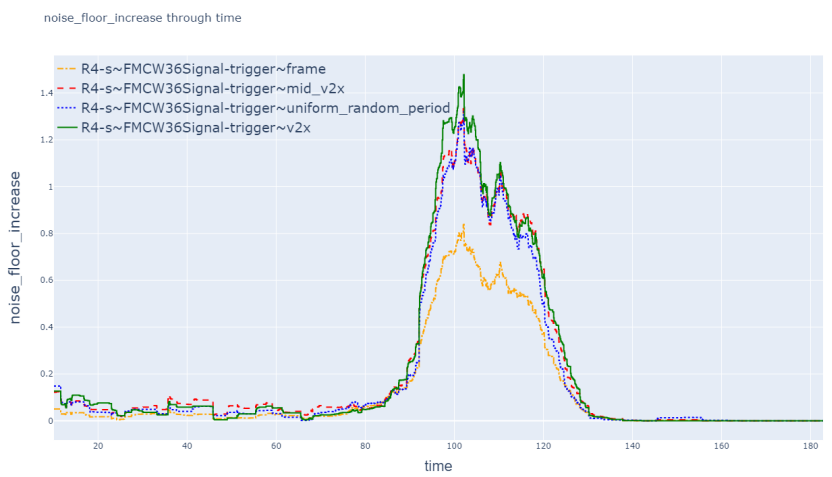

Fig. 8: Average NFI when 36 sets of non interfering parameters are available. At the beginning (and the end) of the simulation, when the two cluster of vehicles are far from each other, few radars are in line-of-sight of each other. As the clusters get closer to each other, more radars start interfering, raising the average noise floor for the victim radar. As the amount of available sets of parameters is relatively large, quickly changing the radar's parameters, even with wrong knowledge of the other radars yields a high probability to stop interfering. That is why the 'Frame' method (yellow), that reacts more quickly to interference than the other three methods has a lower NFI.

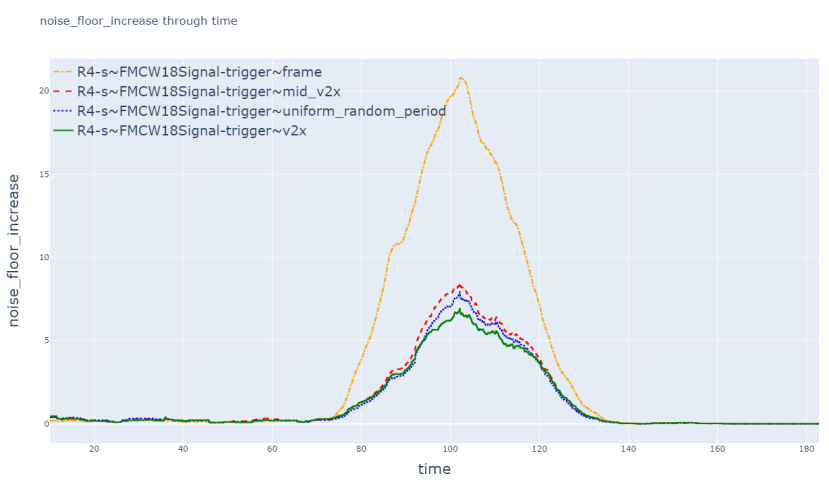

Fig. 9: Average NFI when 18 sets of non interfering parameters are available. With an amount of available sets of parameters halved to 18 , the 'Frame' method (yellow) performs worse than the other three with an average NFI 2-3 times higher as an up-to-date data about other radars' parameters becomes important to make a good parameter choice.

\section{REFERENCES}

[1] "Automotive radar market size, share \& trends analysis report by range (long range, medium \& short range), by vehicle type (passenger cars, commercial vehicles), by application, by frequency, by region, and 6

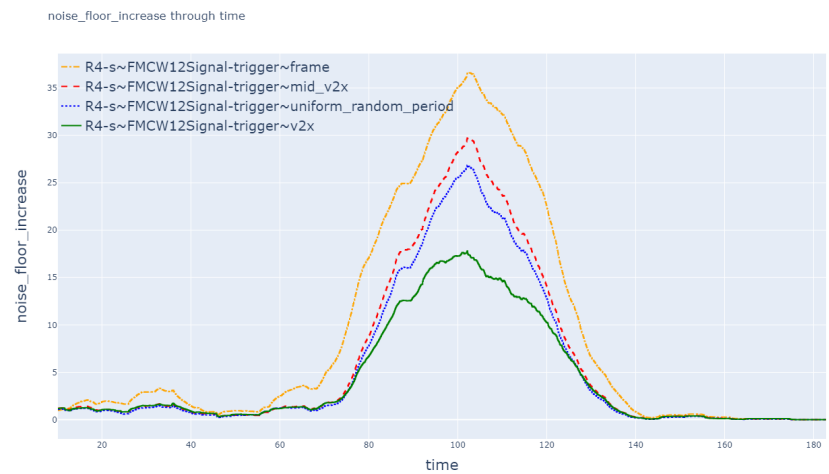

Fig. 10: Average NFI when 12 sets of non interfering parameters are available. As the amount of available sets of parameters is much smaller than the amount of potential interferers in line-ofsight, it is necessary to coordinated the use of the available set of parameters. Using the 'V2X' method (green), that minimizes the delay between parameter choices and CAM emission greatly increases the probability for other radars to be aware of your changes when changing their own parameters. The 'V2X' method is thus yielding the lowest NFI, followed by 'Mid-Interval-V2X' (red) and 'PUR' (blue) than both have an average delay between changes and communication of half a CAM period.

segment forecasts, 2018 - 2025," Grand View Research, Tech. Rep., August 2017.

[2] M. Kunert and R. Bosch, "More safety for all by radar interference mitigation - final report," MOSARIM, Tech. Rep., December 2012.

[3] "Compatibility of automotive collision warning short range radar operating at $79 \mathrm{ghz}$ with radiocommunication services," Electronic Communications Committee (ECC), Tech. Rep., October 2004.

[4] S. Roudiere, V. Martinez, and D. Delahaye, "A first investigation of V2X communication for radar interference mitigation," ITS World Congress 2021, October 2021.

[5] Forbes, "Volkswagen adds 'vehicle-to-everything' communications to revamped golf with nxp chips," October 2019, accessed: 2021-01-15. [Online]. Available: https://www.forbes.com/sites/samabuelsamid/2019/10/28/ volkswagen-includes-nxp-v2x-communications-in-8th-gen-golf/

[6] C-Roads, "C-ITS pioneering demonstration in austria," April 2019, accessed: 2021-01-15. [Online]. Available: https://www.c-roads.eu/platform/about/news/News/entry/show/ c-its-pioneering-demonstration-in-austria.html

[7] EN 302 637-2, Intelligent Transport Systems (ITS); Vehicular Communications; Basic Set of Applications; Part 2: Specification of Cooperative Awareness Basic Service, ETSI Std., April 2019.

[8] D. G. Luck, Frequency modulated radar. McGraw-Hill, 1962.

[9] H. Griffith, "New ideas in fm radar," Electronics \& Communication Engineering Journal, vol. 2, pp. 185-194, 1990.

[10] H. Gu, A. Adrian, V. Martinez, A. Filippi, and K. Moerman, "Analysis of camp report on the C-V2X performance assessment project," 2020. [Online]. Available: https://www.nxp.com/docs/en/white-paper/ CAMPCV2XWP.pdf

[11] 5GAA, "V2X functional and performance test report; test procedures and results," Tech. Rep., April 2019. [Online]. Available: https://5gaa.org/wp-content/uploads/2018/11/5GAA_P-190033_ V2X-Functional-and-Performance-Test-Report_final-1.pdf

[12] A. Bazzi, "LTEV2Vsim network," 2020. [Online]. Available: http://www.wcsg.ieiit.cnr.it/products/LTEV2Vsim.html

[13] A. Bazzi, A. Zanella, I. Sarris, and V. Martinez, "Co-channel Coexistence: Let ITS-G5 and Sidelink C-V2X Make Peace," arXiv:2003.09510 [cs], Mar. 2020, arXiv: 2003.09510. [Online]. Available: http://arxiv.org/abs/2003.09510

[14] V. Martinez and F. Berens, "Survey on ITS-G5 CAM statistics," C2C, Tech. Rep., December 2018. [Online]. Available: https://www.car-2-car.org/fileadmin/documents/General_ Documents/C2CCC_TR_2052_Survey_on_CAM_statistics.pdf 